\title{
Quadrupole Magnetic Center Definition Using the Hall Probe Measurement Technique
}

\author{
Isaac Vasserman \\ Experimental Facility Division, Advanced Photon Source, Argonne National Laboratory
}

\section{Introduction}

The linac coherent light source [LCLS] project [1] requires $5 \mu \mathrm{m}$ straightness of the particle beam trajectory to achieve the desired goal of x-ray multiplication. The main source of beam trajectory distortion is misalignment of quadrupoles. The LCLS project will use a beam-based alignment technique to align the quadrupoles to the needed accuracy. An initial accuracy of the quadrupole alignment not worse than $50 \mu \mathrm{m}$ is required [2]. A different technique could be used for this purpose. It would be though quite desirable to avoid using an additional magnetic measurement technique and to use the same sensors that will be used for undulator magnetic field measurements, i.e., Hall probes. At the APS magnetic measurement facility, an APS FEL quadrupole was used to test this possibility. The results obtained from this test show that an accuracy of $\pm 50 \mu \mathrm{m}$ centerline definition is achievable and allow us to limit the necessary technique to the Hall probe only.

\section{Results of the test}

The ideal quadrupole field dependence on the transverse coordinates is as follows:

$$
\begin{aligned}
& B y=g x \\
& B x=g y
\end{aligned}
$$

where $\mathrm{g}$ is a quadrupole gradient equal in our case to $1.5 \mathrm{kG} / \mathrm{cm}$.

The electromagnetic quadrupole \#11 for the APS FEL project was used for this test. The aperture of the lens is $12 \mathrm{~mm}$, and the longitudinal dimension in iron is $50 \mathrm{~mm}$. The quadrupole does not have side access. A scan was done along the transverse horizontal axis of the bench. The quadrupole was aligned in such a way that the transverse horizontal axis of the bench corresponds to the longitudinal Z-direction of the quadrupole. Probe orientation was used to allow for the horizontal field measurements of the quadrupole. No precise alignment is needed in the direction perpendicular to the Hall plate, because, as is easy to see from the equations above, there is no dependence of the magnetic field in this direction. 
The goal is to find deflection of the quadrupole centerline from the geometrical axis.

Procedure of the test:

1. Alignment of the quadrupole: poles were used as a reference and the quadrupole was aligned with the help of an optical level.

2. Alignment of the probe motion in Z-direction of the quadrupole: clear reference line was made at the probe tip, and the optical level was used for alignment.

3. Zero field location $\mathrm{Y}_{0}$ in the quadrupole center was defined by scanning of the Hall probe in $\mathrm{Y}$ (vertical) direction.

4. Center line scan at $\mathrm{Y}=\mathrm{Y}_{0}$ level in the $\mathrm{Z}$ - direction of the quadrupole was done to define the distortion of the centerline;

Results of the measurement are shown in Fig. 1.

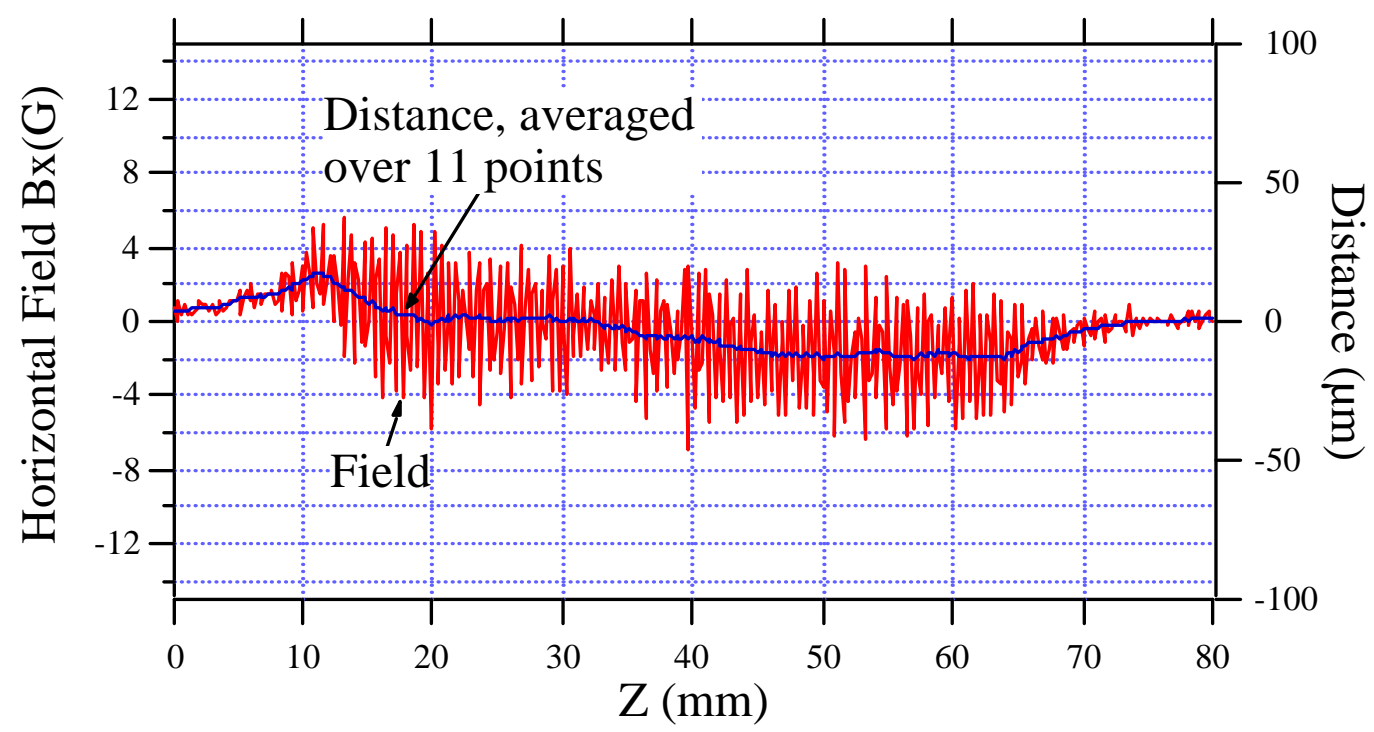

Fig. 1. Horizontal field dependence on the longitudinal direction of the quadrupole lens as measured by the Hall probe.

"Magnetic needles" were used to define the center of the Hall probe sensitive area. These "magnetic needles" were aligned to the same level as the geometrical center of the quadrupole. To eliminate the problems associated with alignment of two needles distance between needles was made big enough and the location of the probe was chosen close to one of the needles. Scanning of the Hall probe in the Y- direction between the needles allowed us to define the center of the Hall probe relative to the geometrical center of the lens.

Results of this scan are shown in Fig. 2. 


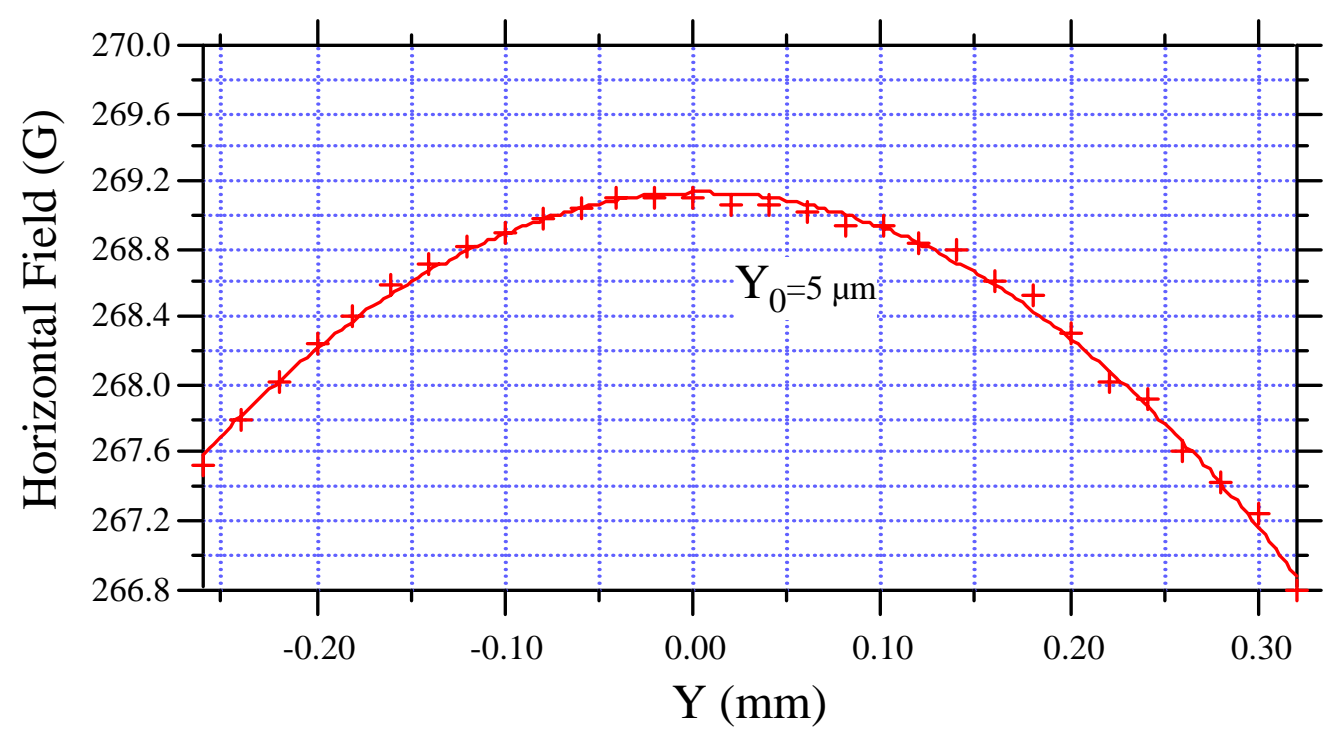

Fig. 2. Location of the Hall probe sensitive area.

The same procedure could be done to define the quadrupole centerline of the vertical field by rotating the lens by $90 \bullet$. This procedure is preferable to rotating the probe and repeating the measurements in another direction. Such measurements are less reliable technically with the equipment we have now for alignment at the APS Magnetic Measurement Facility.

Error sources are:

1. Vertical component of the Z- direction movement of the Hall probe: $\quad \pm 10 \mu \mathrm{m}$

2. Alignment of the quadrupole lens in Z- direction: $\mu \mathrm{m}$

3. Definition of the $\mathrm{Y}_{0}$ of the probe:

$\pm 10 \mu \mathrm{m}$

4. Accuracy of zero field definition by Hall probe scan: $\quad \pm 5 \mu \mathrm{m}$

Assuming the errors as uncorrelated, we can say that the results of these measurements indicate that magnetic and geometrical centerline are in good agreement with accuracy of $\pm 18 \mu \mathrm{m}$.

\section{Conclusions}

The main source of errors is associated with mechanical alignment of the quadrupole, probe and "magnetic needles." The same errors occur for all other types of sensors, except that more attention needs to go into defining the sensitive area of a Hall probe than a pulsed or vibrating wire. Due to the fact that this error is not crucial, the Hall probe is quite adequate to the task. These errors will be essentially the same for LCLS quadrupole measurements. The main conclusion is that we achieved our goal, and Hall probes can be 
used to define the centerline of the quadrupoles for the LCLS project. Another conclusion is that, in our case, the magnetic centerline is in a good agreement with the geometrical one. This conclusion is design related so it is impossible to say the same in advance for the LCLS project quadrupole.

\section{References}

1. LCLS Design Study Report, SLAC-R-521, 1998

2. P. Emma, Beam-Based Alignment of Sector-1 of the SLC Linac, SLAC-PUB-5787, March, 1992 